\title{
Cirugía mínimamente invasiva de la válvula aórtica. 15 años de experiencia en el Hospital Regional de Temuco, Chile.
}

\author{
Juan Carlos Bahamondes. \\ Servicio de Cirugía Cardiovascular, Hospital Regional Temuco, Chile \\ Departamento de Cirugía, Universidad de La Frontera. \\ Temuco, Chile \\ Conflictos de interés: ninguno \\ Financiamento: institucional
}

Introducción: La cirugía de la válvula aórtica puede ser efectuada a través de una esternotomía reducida con menos trauma quirúrgico, una recuperación más rápida, mejores resultados cosméticos y excelentes resultados a largo plazo.

Objetivo: Comunicar los resultados a largo plazo obtenidos con el abordaje mínimamente invasivo en el Hospital Regional de Temuco.

Material y Método: Estudio de cohorte retrospectivo de 286 pacientes operados con técnica mini invasiva comparados con 1161 pacientes operados a través de esternotomía completa para reemplazo valvular aórtico por enfermedad valvular entre 2004 y 2019. La edad promedio fue $62,1 \pm 6,4$ años. Para la canulación arterial y venosa se utilizaron cánulas de tamaño pequeño. El flujo de circulación extracorpórea promedio fue $4.5 \mathrm{~L} / \mathrm{min}$. Se utilizó cardioplejia sanguínea infundida por vía anterógrada y retrograda. Se utilizó estadística descriptiva con medidas de tendencia central y dispersión. Las variables categóricas fueron comparadas con el test de $\mathrm{X}^{\wedge} 2$ y las variables continuas con " $\mathrm{t}$ " test y ANOVA. La supervivencia actuarial se calculó mediante el método de Kaplan Meier con el programa Stata 14.
Resultados: No hubo diferencia en la mortalidad a 30 días entre los grupos. Hallazgos similares se encontraron para arritmias, complicaciones cerebrales y bloqueo AV que requirió marcapasos. Hubo una diferencia estadísticamente significativa a favor del grupo de ministernotomía para reoperación por sangrado, días de estadía en UCI y estadía hospitalaria. El seguimiento promedio fue $101 \pm 4$ ( 1 - 169 meses). $\mathrm{La}$ mayoría de los pacientes estaban en capacidad funcional I y libre de eventos cardiovasculares y reoperación. En el control post operatorio se hizo un ecocardiograma en el $86 \%$ de los pacientes mostrando buen resultado hemodinámico, similar en ambos grupos, y para distintas prótesis utilizadas. La probabilidad de sobrevida actuarial fue de $97 \%$ y la probabilidad de estar libre de eventos cardiovasculares fue $93 \%$ a 156 meses de seguimiento.

Conclusión: La mini esternotomía es una técnico segura para la cirugía valvular aórtica con excelentes resultados en el largo plazo, permitiendo simplificar la cirugía, reducir los costos hospitalarios y permite una recuperación con menos secuelas cosméticas. Palabras clave: miniesternotomía; cirugía valvular aórtica, cirugía mínimamente invasiva. 


\section{Ministernotomy for aortic valve surgery: a 15-year experience.}

Background: Aortic valve surgery can be performed through a reduced ministernotomy with less surgical trauma, improved cosmetics, faster recovery and excellent long-term results.

Aim: To report the long-term results obtained with the minimally invasive approach technique in Southern Chile.

Methods: A retrospective cohort study of 286 patients operated with the less invasive technique compared to 1161 patients operated on with full sternotomy for aortic valve replacement between 2004 and 2019 was performed. Arterial and venous cannulation was performed using small cannulas. Mean extracorporeal circulation flow was $4.5 \mathrm{~L} / \mathrm{min}$. Antegrade and retrograde cold cardioplegia was used. Statistical Analysis: Data are reported with measures of central tendency and dispersion. Categorical variables were compared with the $\mathrm{X}^{\wedge} 2$ test and ANOVA. Actuarial survival rate was calculated by Kaplan Meier method. The Stata 14 package was used for data analysis.

Results: Demographic characteristics are shown in Table 1. Mean age of the whole cohort was 62.1 y/o (SD 6.4). There was no difference in 30 day mortality between groups. Incidence of arrhythmia, cerebral complications and AV block requiring pacemaker was similar between groups. There were no significant statistical differences in reoperation for bleeding, days of stay at the ICU and overall hospital stay. Mean follow up was $101 \pm 4$ (1-169) months. After surgery most patients are in FC I and free from cardiac events and reoperation. In the postoperative period echocardiographic assessment was performed in $86 \%$ of patients showing similar hemodynamic function of the implanted valve. Actuarial survival rate was $97 \%$ and probability of freedom from cardiac events was $93 \%$ at 156 months of follow up. Conclusions: Ministernotomy is an excellent approach for aortic valve surgery which can be performed with very good results not inferior to those obtained with full sternotomy thus simplifying the surgical technique and reducing local hospital costs. Keywods: mini-sternotomy; aortic valve, surgery; minimally invasive surgery. 


\section{Introducción:}

La cirugía mínimamente invasiva de la válvula aórtica fue descrita a principios de la década de los 90 para luego ser ampliamente desarrollada como una alternativa a la esternotomía convencional en pacientes con patología aislada de la válvula aórtica, aorta ascendente y sin compromiso de arterias coronarias ${ }^{1-6}$. Se han descrito una variedad de técnicas quirúrgicas, pero hoy en día se utiliza mayormente la ministernotomía superior, la que ha demostrado resultados favorables en términos de un menor sangrado y dolor postoperatorio, una menor estadía hospitalaria permitiendo una disminución en los costos de la cirugía valvular aórtica, y una incisión pequeña con mejores resultados cosméticos sin variación en la supervivencia de los pacientes $7,8,9,10$.

La cirugía mini invasiva de la válvula aórtica requiere de nuevas habilidades en el arsenal técnico del cirujano y la curva de aprendizaje asociada es importante ${ }^{11,12}$. Lo que para algunos puede ser disuasivo, para muchos grupos de cirugía cardíaca ha sido un reto que ha permitido su desarrollo, demostrando sus ventajas en las casi 3 décadas desde sus inicios $13,14,15,16,17,18$.

\section{Pacientes y Método:}

Entre junio de 2004 y Junio 2019 se realizó reemplazo valvular aórtico en 1447 pacientes consecutivos en forma electiva. En 286 pacientes se accedió al corazón mediante una miniesternotomía media. Los 1161 pacientes restantes fueron intervenidos mediante esternotomía media convencional y constituyen el grupo control. La decisión de utilizar la miniesternotomía fue efectuada por el cirujano, quien seleccionó a pacientes mesomórficos sin distingo por edad o por predominio de estenosis o insuficiencia aórtica, y fue progresiva más frecuente en el tiempo a medida que los resultados de la técnica eran favorables.

\section{Método:}

Estudio retrospectivo de cohortes en quienes se efectuó reemplazo valvular aórtico mediante miniesternotomía, comparados con pacientes en los que se efectuó esternotomía completa, identificados mediante la base de datos de cirugía cardiovascular de nuestro centro. Se revisaron las fichas clínicas y protocolos operatorios. En el estudio preoperatorio, se realizó ecocardiografía a todos los pacientes y coronariografía en mayores de 50 años. Para el seguimiento se utilizaron datos de las fichas clínicas, controles en policlínico, datos obtenidos en el registro civil, o mediante contacto telefónico con el paciente.
Técnica quirúrgica. Nuestra técnica fue descrita en un estudio previo. Previa inducción de la anestesia general, el paciente fue posicionado en decúbito supino instalando parches autoadhesivos para desfibrilación externa en la región dorsal. El campo quirúrgico fue preparado con técnica estéril en forma habitual. Se accedió al tórax mediante una incisión cutánea en la línea media no mayor a 8-10 cm, ubicando el ángulo de Louis y luego midiéndose 2 a $3 \mathrm{~cm}$ sobre este punto de reparo hacia la incisura esternal. El resto de la incisión se prolongó hacia el cuerpo esternal.

Luego el esternón fue transectado mediante sierra neumática desde el yugulum hasta el cuarto espacio intercostal. A ese nivel el hueso fue abierto en forma horizontal, teniendo precaución de no dañar la arteria mamaria interna derecha. Posteriormente, el tejido graso y remanente tímico del mediastino anterior fue seccionado y ligado para evitar sangrados posteriores. El pericardio fue abierto en la línea media en forma de T invertida, asegurándolo mediante suturas ajustadas al borde de la incisión, con lo cual se logró una excelente exposición de la aorta ascendente en todo su trayecto como también de la aurícula derecha. Luego de la heparinización sistémica, la circulación extracorpórea (CEC) fue establecida mediante canulación en aorta ascendente o cayado proximal y cánula venosa única de 3 etapas en la aurícula derecha, utilizando hipotermia sistémica moderada hasta $34^{\circ} \mathrm{C}$. El pinzamiento aórtico se realizó mediante un clamp de Cosgrove. Las cavidades izquierdas fueron drenadas mediante un vent aurículo-ventricular introducido a través de la vena pulmonar superior derecha o mediante un aspirador directo en el ventrículo izquierdo a través del anillo aórtico durante el periodo de extracción y aseo valvular. La protección miocárdica se efectuó mediante solución de cardioplejia hemática a $4^{\circ} \mathrm{C}$, infundida en forma intermitente cada 20 min en la raíz aórtica en los casos de predominio de estenosis aórtica y en los ostia coronarios en forma directa, luego de abrir la aorta en los pacientes en que predominó la insuficiencia aórtica. Aunque no de forma rutinaria, se empleó cardioplejia retrograda a través de la aurícula derecha en el seno coronario. En todos los pacientes se realizó aortotomía oblicua permitiendo una excelente exposición de la válvula aórtica y ostia coronarios mediante suturas de tracción de las comisuras. La válvula fue extraída completamente, decalcificando luego el anillo aórtico. La prótesis de elección fue suturada en el anillo mediante suturas de Tycron 2/0 apoyadas sobre pledgets de teflón, visualizando ambos ostia coronarios al completar el procedimiento. La aortotomía 
fue cerrada con sutura continua no reabsorbible 4/0. Se removió el aire de las cavidades y se procedió al despinzamiento aórtico y retiro de CEC. Luego de revisada la hemostasia, se instalaron cables de marcapaso epicárdicos y uno o más drenajes mediastínicos en la línea media.

Estadística: Se utilizó estadística descriptiva con medidas de tendencia central y dispersión; test exacto de Fisher y chi2 de Pearson para variables dicotómicas y t-test $o$ análisis de varianza para variables continuas. Se consideró estadísticamente significativo un valor $\mathrm{p}<0,05$. Se recurrió al método de Kaplan-Meier para el cálculo de supervivencia actuarial Se utilizó Stata $14{ }^{\circledR}$ para el análisis de datos.

\section{Resultados:}

La edad promedio incluidos todos los pacientes fue 62,1 años $(\mathrm{DE} \pm 6,4)$ con un rango entre 32 a 89 años. La distribución por sexo fue similar. En el período preoperatorio la mayoría de los pacientes estaban en CF III, según la clasificación NYHA (Tabla 1).

En todos los casos efectuados a través de miniesternotomía se consiguió una buena exposición valvular lo que permitió el reemplazo protésico sin contratiempos. El acceso esternal varió entre 8 y $10 \mathrm{~cm}$, con un promedio de $9 \mathrm{~cm}$. El tiempo de CEC fue en promedio de $67 \mathrm{~min}$ (DE $\pm 4,75)$ y el de clamp fue $46 \min (\mathrm{DE} \pm 4,65)$, respectivamente, alcanzando un flujo de CEC de 4,5 L/min en promedio, sin diferencias estadísticamente significativas con el grupo control (Tabla 1). La estadía postoperatoria en Unidad de Cuidados Intensivos (UCI) y la cantidad de días de hospitalización fue menor en el grupo intervenido mediante miniesternotomía, alcanzando significancia estadística. Las reoperaciones por sangrado fueron me-
Tabla 1. Variables biodemográficas de pacientes intervenidos por Miniesternotomia y Esternotomía convencional

\begin{tabular}{|c|c|c|c|c|c|}
\hline Variable & \multicolumn{2}{|c|}{$\mathrm{n}=286$} & \multicolumn{2}{|c|}{$n=1161$} & $p$ \\
\hline Edad promedio & \multicolumn{2}{|c|}{$61.3 \pm 15.2$} & \multicolumn{2}{|c|}{$63.1 \pm 17.6$} & $p=0.76$ \\
\hline Sexo Masculino(\%) & \multicolumn{2}{|c|}{$64 \%$} & \multicolumn{2}{|c|}{$67 \%$} & $\mathrm{p}=0.59$ \\
\hline \multirow[t]{4}{*}{ Capacidad Funcional } & & 0 & & & \\
\hline & & 87 & II & 297 & $p=0.64$ \\
\hline & & 196 & III & 852 & $p=0.73$ \\
\hline & IV & & IV & 12 & $p=0.45$ \\
\hline Tiempo CEC & \multicolumn{2}{|c|}{$67.2 \pm 4.75$} & \multicolumn{2}{|c|}{$67.6 \pm 15.7$} & $p=0.78$ \\
\hline Tiempo Clamp & \multicolumn{2}{|c|}{$46 \pm 4.65$} & \multicolumn{2}{|c|}{$53 \pm 12.4$} & $p=0.83$ \\
\hline
\end{tabular}

nores en el grupo estudio $(\mathrm{p}<0.05)$. El sangrado postoperatorio y el número de transfusiones fueron menores para la miniesternotomía. No hubo conversión a esternotomía completa en ningún caso ni infección esternal profunda. No hubo diferencias en arritmias, accidente cerebral y bloqueo $\mathrm{AV}$ que requiriese marcapasos definitivo entre los grupos. Tampoco hubo diferencia en la mortalidad a 30 días (Tabla 2).

La distribución de los 286 pacientes operados con miniesternotomia según tipo de prótesis utilizada e índice de área de orificio efectivo (IAOE) se muestra en la Tabla 3. Se observa que existió una mayor cantidad de pacientes con prótesis mecánicas y de pericardio bovino con un IAOE adecuado. El porcentaje de desproporción prótesis/paciente fue mayor para los pacientes con prótesis biológicas de porcino, por lo que en nuestro centro se descontinuó su utilización cambiando a prótesis de pericardio bovino (a discusión).

\begin{tabular}{|l|c|c|c|}
\hline \multicolumn{4}{|c|}{$\begin{array}{c}\text { Tabla 2. Comparación de pacientes intervenidos con } \\
\text { Miniesternotomía y Esternotomía Convencional. }\end{array}$} \\
\hline Variable & $\begin{array}{c}\text { Mini Esternotomía } \\
(n: 286)\end{array}$ & $\begin{array}{c}\text { Esternotomía Convencional } \\
(\mathrm{n}: 1161)\end{array}$ & $\mathrm{p}$ \\
\hline Mortalidad <30 días & $4(1,4 \%)$ & $14(1,21 \%)$ & $\mathrm{p}=0,4$ \\
\hline Arritmias & $45(15,7 \%)$ & $279(24 \%)$ & $\mathrm{p}=0,38$ \\
\hline Complicaciones Cerebrales & $5(1,7 \%)$ & $23(2 \%)$ & $\mathrm{p}=0,52$ \\
\hline Bloqueo AV /Marcapasos & $23(8 \%)$ & $151(13 \%)$ & $\mathrm{p}=0,06$ \\
\hline Reoperación Sangrado & $5(1,8 \%)$ & $35(3 \%)$ & $\mathrm{p}=0,03$ \\
\hline Estadía ICU & $2,2 \pm 1,4$ & $3,8 \pm 2,6$ & $\mathrm{p}=0,04$ \\
\hline Estadía Hospital & $4,2 \pm 1,7$ & $6,7 \pm 1,4$ & $\mathrm{p}=0,05$ \\
\hline
\end{tabular}




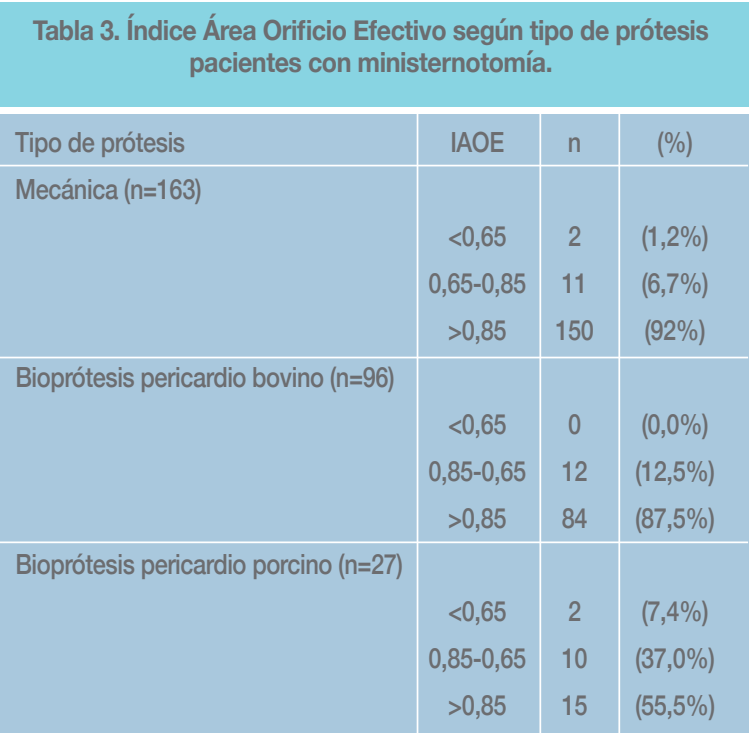

Seguimiento. El seguimiento promedio fue de 156,3 (DE $\pm 4,25$ ) meses ( 1 a 169 ) y pudo completarse en $88 \%$ de los casos debido a perdida de controles y mortalidad por otras causas a 15 años. La probabilidad de supervivencia actuarial fue $97 \%$ y de la probabilidad libre de eventos cardiovasculares fue $93 \%$.

La gran mayoría de los pacientes se encuentran en CF I y libres de eventos cardiovasculares en el período observado. Se realizó ecocardiografía de control en el $78 \%$ de los pacientes, evidenciándose que en el caso de prótesis mecánicas había una buena movilidad de los discos protésicos, y no hubo daño estructural en los velos de las válvulas biológicas a 6 años de instalación. Tampoco hubo deterioro de la función ventricular y no se demostró derrame pericárdico. Durante este período no se observó ninguna secuela estética derivada de la cicatriz de la herida operatoria y si bien no se utilizó ninguna escala de satisfacción del procedimiento, éste fue catalogado como de excelente resultado estético por la mayoría de los pacientes.

\section{Discusión:}

Los beneficios de la cirugía de la válvula aórtica a través de una incisión reducida han sido ampliamente difundidos, pero existe aún escepticismo en aquellos que buscan una mayor evidencia ${ }^{11,19,20,21,22,23}$. En la literatura internacional se describe ampliamente que la miniesternotomía produce un menor trauma quirúrgico que se traduce en menor dolor postoperatorio y una recuperación más rápida, minimizando las complicaciones derivadas de la esternotomía media $3,5,6,7,11,14,17,19,24,25,26$.
Después de una larga experiencia con la esternotomía convencional, desde el año 2004 comenzamos nuestra serie de miniesternotomía en $J$ en forma progresiva ya que también permite una visualización directa de la aorta y estructuras cardíacas derechas, y que, junto con el advenimiento de cánulas más pequeñas, han permitido reducir aún más el tamaño de la incisión, con un riesgo quirúrgico similar en comparación con el grupo de esternotomía clásica ${ }^{27}$.

En nuestro medio algunos grupos han demostrado excelentes resultados ${ }^{27,28}$ con incisiones de longitud similar a la expuesta en la literatura y en nuestro centro, sin necesidad de convertir a esternotomía media. A nivel mundial, algunos estudios demuestran que para reemplazo aislado de la válvula aórtica, existe un tiempo menor en ventilación mecánica, una estadía menor en UCI y días de hospitalización y con sobrevidas a mediano y largo plazo comparables con los pacientes operados con esternotomía clásica. Actualmente, numerosos estudios han demostrado mejores resultados intra hospitalarios como disminución del dolor post operatorio, facilitando así una mejor mecánica pulmonar y movilización pre$\operatorname{coz}^{7,11,14,19}$.

Otras ventajas, que demuestran grupos con gran experiencia en cirugía mínimamente invasiva, son un menor sangrado con la consiguiente ventaja de menor uso de transfusiones que en el grupo control ${ }^{11,14,17,19,25,27}$. En nuestra serie se objetivó este hecho y menor operación por esta causa, coincidiendo con un uso significativamente menor de transfusiones en el período postoperatorio. Esto demuestra un menor trauma quirúrgico en estos pacientes. Otros estudios corroboran que existe un mayor sangrado en las primeras horas para el grupo de esternotomía convencional y mayor índice de operación, hallazgo similar al encontrado en nuestra serie $5,7,11,14,19,25$, En relación a la presencia de un nuevo episodio de fibrilación auricular entre ambas técnicas, varios estudios demuestran ventajas a favor del grupo de miniesternotomía $29 \cdot 30,31,32,33$. En nuestra serie no encontramos diferencias significativas, aunque se observa que existe una clara menor presencia de FA en pacientes con miniesternotomía. Otro hecho interesante es que el bloqueo AV y necesidad de marcapasos definitivo fue significativamente menor en el grupo de miniesternotomía. Otros estudios que comparan ministernotomía con mini toracotomía han demostrado aún mejores resultados en relación a presencia de FA y una menor estadía hospitalaria, pero faltan estudios con mayor número de pacientes para poder soportar una u otra técnica ${ }^{34,35,36}$. La estadía hospitalaria fue 
similar a otras publicaciones, fluctuando entre 4 y 6 días, existiendo diferencias estadísticamente significativas a favor de la ministernotomía. Además, hasta el momento no ha sido necesaria la conversión a esternotomía completa en ningún paciente $7,11,12,17,19,24,27,30$. En relación a seguimiento y supervivencia a largo plazo y necesidad de operación, no encontramos diferencias estadísticamente significativas entre los grupos ${ }^{11,14,25}$.

En base a lo anterior, se puede concluir que la experiencia alcanzada en forma progresiva con la miniesternotomía en J utilizada para la cirugía de la válvula aórtica esta es una excelente alternativa de acceso, consiguiendo un campo operatorio satisfactorio, manteniendo la eficiencia y eficacia al permitir una completa visualización de la raíz y aorta ascendente con una reducción en el trauma- tismo esternal y sin producir un aumento en el tiempo quirúrgico total. Los avances en la técnica quirúrgica en sí y en el post operatorio de estos pacientes, asociado a una movilización activa y precoz, han permitido disminuir los requerimientos de transfusión, estadía en UCI y hospitalaria sin comprometer la supervivencia en el largo plazo 11,25,30,31,32. Además, esta técnica no sólo provee resultados cosméticos satisfactorios para los pacientes, sino que además posibilita una recuperación precoz y con secuelas mínimas.

Como limitaciones deben mencionarse la falta de aleatorización y el carácter retrospectivo del estudio. Así mismo, el hecho de que los criterios de inclusión, determinados por el cirujano, fueron necesariamente influidos por características de los pacientes.

\section{Referencias}

1. JULIÁN OC, LÓPEZ-BELIO M, DYE WS. The median sternal incision in intracardiac surgery with extracorporeal circulation: a general evaluation of its use in heart surgery. Surgery 1957; 42: 753-61.

2. COSGROVE III DM, SABIR JF. Minimally invasive approach for aortic valve operations. Ann Thorac Surg 1996; 62: 596-7.

3. COSGROVE III DM, NAVIA JL, SABIR JF. Minimally invasive valve operations. Ann Thorac Surg 1998; 65: 1535-9.

4. SVENSSON LG, D'AGOSTINO RS. "J" incisión minimal-access valve operations. Ann Thorac Surg 1998; 66: 1110-2.

5. SVENSSON LG, D'Agostino RS. Minimal-access aortic and valvular operations, including the "J" incisión. Ann Thorac Surg 1998; 66: 431-5.

6. RAO PN, KUMAR AS. Aortic valve replacement through right thoracotomy. Tex Heart Inst J 1993;20:307-8.

7. COHN LH, ADAMS DH, COUPER GS, et al. Minimally invasive cardiac valve surgery improves patient satisfaction while reducing costs of cardiac valve replacement and repair. Ann Surg 1997;226:421-6; discussion 427-8.

8. BENETTI FJ, MARIANI MA, RIZZARDI JL, et al. Minimally invasive aortic valve replacement. J Thorac Cardiovasc Surg 1997;113:806-7.

9. VON SEGESSER LK, WESTABY S, POMAR J, et al. Less invasive aortic valve surgery: rationale and technique. Eur J Cardiothorac Surg 1999;15:781-5.

10. MORENO-CABRAL RJ. MINI-T sternotomy for cardiac operations. J Thorac Cardiovasc Surg 1997;113:810-1.

11. Minimally Invasive Approaches to Surgical Aortic Valve Replacement: A Meta-Analysis. Chang C, Raza S, Altarabsheh SE, Delozier S, Sharma UM, Zia A, Khan MS, Neudecker M, Markowitz AH, Sabik JF 3rd, Deo SV. Ann Thorac Surg. 2018 Dec;106(6):1881-1889. doi: 10.1016/j.athoracsur.2018.07.018. Epub 2018 Sep 4.

12. BONACCHI M, PRIFTI E, GIUNTI G, FRATI G, SANI G. 
Does ministernotomy improve postoperative outcome in aortic valve operation? A prospective randomized study. Ann Thorac Surg 2002; 73: 460-5.

13. ARIS A, CÁMARA ML, MONTIEL J, DELGADO LJ, GALÁN J, LITVAN H. Ministernotomy versus median sternotomy for aortic valve replacement: a prospective, randomized study. Ann Thorac Surg 1999; 67: 1583-7; discussion 1587-8

14. COHN LH, ADAMS DH, COUPER GS. Minimally invasive cardiac valve surgery improves patient satisfaction while reducing costs of valve replacement and repair. Ann Surg 1997; 226: 421-6.

15. WEINSCHELBAUM E, STUTZBACH P, MACHAIN A, FAVALORO R, CARAMUTTI V, BERTOLOTTI A, FRAGUAS H. Valve operations through a minimally invasive approach. Ann Thorac Surg 1998; 66: 1106-9.

16. ARIS A, PADRÓ JM, Cámara ML. Sustitución valvular aórtica mínimamente invasiva. Rev Esp Cardiol 1997; 50: 750-3.

17. COOLEY D. Minimally invasive valve surgery versus the conventional approach. Ann Thorac Surg 1998; 66: 1101-5.

18. SZWERC MF, BENCRART DH, WIECHMANN RJ, SAVAGE EB, SZYDLOWSRI GW, MAGOVERN JR GJ, MAGOVERN JA. Partial versus full sternotomy for aortic valve replacement. Ann Thorac Surg 1999; 68: 2209-13.

19. GILMANOV D, BEVILACQUA S, MURZI M, et al. Minimally invasive and conventional aortic valve replacement: a propensity score analysis. Ann Thorac Surg 2013;96:837-43.

20. DOGAN S, DZEMALI O, WIMMER-GREINECKER G, et al. Minimally invasive versus conventional aortic valve replacement: a prospective randomized trial. J Heart Valve Dis 2003;12:76-80.

21. SHARONY R, GROSSI EA, SAUNDERS PC, et al. Minimally invasive aortic valve surgery in the elderly: a case-control study. Circulation 2003;108Suppl 1:II43-7.

22. BAKIR I, CASSELMAN FP, WELLENS F, et al. Minimally invasive versus standard approach aortic valve replacement: a study in 506 patients. Ann Thorac Surg 2006;81:1599-604.

23. BONACCHI M, PRIFTI E, GIUNTI G, et al. Does ministernotomy improve postoperative outcome in aortic valve operation? A prospective randomized study. Ann Thorac Surg 2002;73:460-5; discussion 465-6.

24. MÄCHLER HE, BERGMANN P, ANELLI-MONTI M, et al. Minimally invasive versus conventional aortic valve operations: a prospective study in 120 patients. Ann Thorac Surg
$1999 ; 67: 1001-5$.

25. BROWN ML, MCKELLAR SH, SUNDT TM, et al. Ministernotomy versus conventional sternotomy for aortic valve replacement: a systematic review and meta-analysis. J Thorac Cardiovasc Surg 2009;137:670-9.

26. MIHALJEVIC T, COHN LH, UNIC D, et al. One thousand minimally invasive valve operations: early and late results. Ann Surg 2004;240:529-34; discussion 534.

27. BAHAMONDES S, JUAN CARLOS, MERIÑO S GUSTAVO, SALMAN A JUAN, SILVA V ABELARDO, DROGUETT G JEAN PIERRE. Miniesternotomia para cirugia valvular aórtica. Experiencia inicial en un centro cardiovascular en Chile. Rev Méd Chile 2008; 136: 1141-1146

28. ZALAQUETT R, BAEZA C, IRARRÁZAVAL M, MORÁN S, BECRER P, MATURANA G et al. Esternotomía mínima en cirugía valvular. Rev Chil Cardiol 1999; 18: 63-8

29. MALAISRIE SC, BARNHART GR, FARIVAR RS, et al. Current era minimally invasive aortic valve replacement: techniques and practice. J Thorac Cardiovasc Surg 2014;147:6-14.

30. DEWEY TM, HERBERT MA, RYAN WH, et al. Influence of surgeon volume on outcomes with aortic valve replacement. Ann Thorac Surg 2012;93:1107-12; discussion 1112-3.

31. GOSEV I, KANEKO T, MCGURK S, et al. A 16-year experience in minimally invasive aortic valve replacement: context for the changing management of aortic valve disease. Innovations (Phila) 2014;9:104-10; discussion 110.

32. LIU J, SIDIROPOULOS A, KONERTZ W. Minimally invasive aortic valve replacement (AVR) compared to standard AVR. Eur J Cardiothorac Surg 1999;16Suppl 2:S80-3.

33. HOGUE CW, JR, HYDER ML. Atrial fibrillation after cardiac operation: risks, mechanisms, and treatment. Ann Thorac Surg 2000;69:300-6.

34. ITO T, MAEKAWA A, HOSHINO S, et al. Right infraaxillary thoracotomy for minimally invasive aortic valve replacement. Ann Thorac Surg 2013;96:715-7.

35. MICELI A, MURZI M, GILMANOV D, et al. Minimally invasive aortic valve replacement using right minithoracotomy is associated with better outcomes than ministernotomy. J Thorac Cardiovasc Surg 2014;148:133-7.

36. GLAUBER M, MICELI A, GILMANOV D, et al. Right anterior minithoracotomy versus conventional aortic valve replacement: a propensity score matched study. J Thorac Cardiovasc Surg 2013;145:1222-6. 